\title{
Lesões causadas por alguns helmintos em galinhas-d'angola (Numida meleagris, L.) procedentes do estado do Rio de Janeiro
}

\section{Lesions due to some helminths in guinea fowls (Numida meleagris, L.) from the State of Rio de Janeiro}

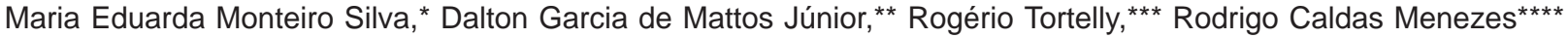

\begin{abstract}
Resumo
Um estudo descritivo das lesões causadas por helmintos dos gêneros: Ascaridia, Paratanaisia e Raillietina que acometem as criações extensivas de galinha-d'angola no estado do Rio de Janeiro, foi realizado no período de janeiro de 2002 a agosto de 2003. A pesquisa baseou-se no exame clínico ante-mortem, eutanásia e coleta de material destinado à histopatologia de 22 aves. Nestas, foram abordados os aspectos macro e microscópicos das injúrias, considerando a intensidade média de infecção e a lesão determinada pelos gêneros dos helmintos estudados. Dos aspectos abordados, no presente trabalho, a enterite crônica com áreas de destruição da mucosa foi a lesão que apresentou, concomitantemente com as alterações macroscópicas, maior significado, sendo esta causada por parasitas do gênero Raillietina. O gênero Ascaridia, estava associado a uma enterite parasitária com discreta reação inflamatória e o gênero Paratanaisia, a uma discreta reação inflamatória peritubular associada à dilatação dos ductos coletores.
\end{abstract}

Palavras-chave: galinha-d'angola, helmintos, lesões, Raillietina magninumidae, Ascaridia numidae, Tanaisia bragai.

\begin{abstract}
A pathology descriptive of the injury caused by: Ascaridia numidae, Tanaisia bragai e Raillietina magninumiade which occur in free range reared guinea fowls in the state of Rio de Janeiro, was carried out during the period from january 2002 until august 2003. Results were establish in the clinical ante-mortem, necropsies and tissue samples for histopathology examination of 22 guinea fowls. In this study, was approached the macroscopic and microscopic lesions, considering the intensity of infection, and the injury determined by each helminths was analyzed. At present work, the intestinal chronic enteritis with areas of the destruction of mucous membrane, was the most important injury, concomitant which the macroscopics changes caused by $R$. magninumidae. A. numidae, was associate a intestinal parasitic chronic enteritis with discreet inflammatory reaction, and $T$. bragai, determined a discreet inflammatory reaction and dilatation of the renal collecting ducts where the trematodes were found.
\end{abstract}

Keywords: guinea fowl, helminths, lesions, Raillietina magninumidae, Ascaridia numidae, Tanaisia bragai.

\section{Introdução}

A galinha-d'angola (Numida meleagris) é uma ave pertencente à ordem Galliformes e família Phasianidae como as galinhas, perus e faisões. É nativa da África Ocidental, onde constitui uma importante fonte econômica para diversas comunidades deste continente (Menezes, 2001).

Foi introduzida no Brasil pelos colonizadores portugueses. A França e a Rússia são, atualmente, os principais países consumidores da carne de galinha-d'angola.

No Brasil, a criação desta ave tem sido uma alternativa econômica que vem atraindo cada vez mais criadores interessa- dos em abastecer hotéis e restaurantes com sua carne, bem como fornecê-las para ornamentações de sítios e fazendas. A ave se adaptou tão bem no País, que pode ser encontrada desde os pampas gaúchos até o nordeste. Na cidade de Fortaleza (CE), encontra-se um dos maiores plantéis comerciais de galinha-d'angola do país. A Emape Alimentos S.A. mantém uma criação com 10 mil matrizes e produz, semanalmente, 8 mil pintos, que são vendidos, para engorda, a criadores de vários Estados (Spínola, 1997).

As afecções parasitárias das aves promovem perdas, sobretudo em animais jovens, segundo avalia Euzéby (1961) e caracteriza-se como uma relação estreita com seu hospedeiro

\footnotetext{
* MS, Ornitopatologia UFF. Rua Cinco de Julho, 486, apto. 403, Santa Rosa, Niterói, RJ, CEP 24220-111.memsilva@bol.com.br.

** MS, PhD, Professor do Departamento de Saúde Coletiva Veterinária e Saúde Pública da Faculdade de Veterinária -- Universidade Federal Fluminense (UFF) - Rua Vital Brazil Filho, 64, Niterói, RJ, Brasil, CEP 24220-050. Dgmjrvet@vm.uff.br.

${ }^{* * *}$ Professor do Departamento de Patologia e Apoio Clínico da Faculdade de Veterinária da Universidade Federal Fluminense (UFF) - Rua Vital Brazil Filho, 64, Niterói, RJ, Brasil, CEP 24220-050.

${ }^{* * * \star}$ Médico-veterinário do Departamento de Controle de Qualidade Animal - Centro de Criação de Animais de Laboratório da FIOCRUZ.
} 
vertebrado (López et al., 1999). Diante deste conceito, o surgimento de problemas sanitários num plantel requer o correto diagnóstico, dentro de um menor tempo possível, para que o lote volte a produzir o esperado.

Segundo Barger et al. (1959), as aves domésticas são susceptíveis de serem infectadas por grande número de parasitas internos e poucos lotes de aves estariam totalmente livres. A interferência nas funções do complexo mecanismo fisiológico do hospedeiro, é o que conduz ao dano celular e tissular, causando lesões nos órgãos parasitados, refletindo, assim, na queda da produtividade e até na mortalidade das aves doentes, interferindo nas perdas econômicas (Boado et al., 1991; López et al., 1999).

Foram estudados, na presente pesquisa, os aspectos anátomo e histopatológico das lesões causadas pelos parasitas buscando a correlação com a intensidade da infecção. Tais fatos podem ser de grande valia para o diagnóstico clínico numa criação, proporcionando condições para medidas de controle parasitário voltadas para uma melhor qualidade de vida em uma criação extensiva direcionada para a comercialização.

\section{Material e métodos}

Foram utilizadas 22 galinhas-d'angola pertencentes a diversas faixas etárias, oriundas de criações extensivas situadas em diversos municípios do estado do Rio de Janeiro: Cambuci, Corrêas, Itaboraí, Petrópolis e São Francisco de Itabapuana.

Antes da necrópsia, foi feito um exame externo, onde cada ave foi avaliada clinicamente, através da inspeção de pele, penas, olhos, mucosas e excrementos e também pela observação da coloração da musculatura peitoral.

As aves foram registradas no Laboratório de Doenças Parasitárias e no Serviço de Anatomia Patológica da Faculdade de Veterinária da Universidade Federal Fluminense (UFF). Foram coletados dados como idade (tendo como parâmetro o tamanho das cristas), sexo, procedência. As amostras de fezes foram coletadas para a realização de exames parasitológicos para pesquisa de ovos e oocistos, através das técnicas de Gordon e Whitlock, segundo Umeche e Eno (1987) e Ueno e Gonçalves(1994).

Em seguida os animais foram submetidos ao método convencional de necrópsias segundo Zander e Mallinson (1991) e Lucio-Martínez (1999).

$\mathrm{Na}$ necrópsia foram retirados fígado, rim e intestinos, e os seus conteúdos foram colocados em placa de Petri estéril contendo solução salina $(0,85 \%)$ e com auxílio do estereoscópio, procedeu-se à análise dos mesmos, e avaliação macroscopicamente quanto a coloração, consistência, aspecto, volume e presença de helmintos ao corte.

Os fragmentos, destinados à histopatologia, foram fixados em formol a $10 \%$ e enviados para o Laboratório de Anatomia Patológica da Faculdade de Veterinária da UFF, onde foram processados pelas técnicas de inclusão em parafina, corados pela hematoxilina -eosina (HE) e montados de acordo com as técnicas histológicas usuais segundo Behmer et al. (1976), para evidenciação das lesões.
No estudo microscópico levou-se em consideração a relação entre o número de parasitas e a injúria causada, bem como o caráter patogênico do parasita; a intensidade média de infecção encontrada e extensão da lesão.

Os intestinos e rins foram lavados com salina estéril $(0,85 \%)$ em placa de Petri para remoção dos parasitas e posteriormente contá-los e idetificá-los (Umeche e Eno, 1987).

Os helmintos encontrados foram coletados e fixados em solução contendo: $93 \%$ álcool, $70^{\circ} \mathrm{GL}, 5 \%$ formol comercial e $2 \%$ ácido acético (A.F.A.), contados e identificados de acordo com as técnicas helmintológicas, segundo Wardle e McLoad (1952), Yamaguti (1961), Levine (1980), Thatcher (1993) e Vicente et al. (1995).

Exemplares de nematóides do gênero Ascaridia, foram fixados pelo AFA a quente, e em seguida clarificados pelo lactofenol de AMAN (Amato et al., 1990), e provisoriamente montados entre lâmina e lamínula, e identificados segundo Yamaguti (1961), Costa e Freitas (1962), Levine (1980), Ruff (1991) e Vicent et al. (1995).

Os cestóides e trematódeos encontrados, foram submetidos à técnica de coloração pelos carmins (Pinto, 1945), tendo sido utilizado como corante a solução de bórax-carmin preparada de acordo com Ueno e Gonçalves (1994).

Uma vez corados e clarificados, esses parasitas foram montados em bálsamo do Canadá entre lâmina e lamínula. Posteriormente, os cestóides foram identificados segundo Wardle e McLeod (1952), Reid (1962) e Freitas e Costa (1962) e os trematódeos de acordo com Freitas (1962), Travassos et al. (1969) e Thatcher (1993).

\section{Resultados}

De um total de 22 galinhas-d'angola necropsiadas e examinadas, $17(77,27 \%)$ encontravam-se parasitadas por uma ou mais espécies de helmintos. Os nematóides do gênero Ascaridia estavam presentes em 15 aves $(68,20 \%)$, os trematódeos do gênero Paratanaisia em sete $(31,80 \%)$ e os cestóides do gênero Raillietina em cinco $(22,70 \%)$, e em apenas cinco das aves $(22,73 \%)$ não foram observados parasitas.

Dentro dos gêneros estudados, foram identificadas as espécies: Ascaridia numidae (Leiper, 1908), Paratanaisia bragai (Santos, 1934), Raillietina (Paroniella) magninumida (Jones, 1930).

A maioria das galinhas-d'angola examinadas apresentava um grande número de parasitas, sendo que o total de helmintos adultos encontrados foi igual a 2.569, com um número médio de 151,1 helmintos por ave. Em relação ao número total de helmintos dos gêneros Ascaridia, Paratanaisia e Raillietina observados, Ascaridia (1.156) e P. bragai (272 ) foram encontrados em grande número.

Cerca de $55 \%$ das aves infectadas estavam em estado de carne relativamente bom, uma boa cobertura muscular, além de plumagem adequada, cristas e barbelas normocoradas, e o restante das aves apresentavam caquexia, caracterizada por atrofia muscular - fato observado na musculatura peitoral; plumagens eriçadas e sem brilho. Apenas uma ave apresentou sinuosidade do esterno.

Na Tabela 2 está relacionado o estado de carne em relação a cada parasita estudado. 
Tabela 1 - Helmintoses em galinhas-d'angola de alguns municípios do estado do Rio de Janeiro - 2003

\begin{tabular}{lccccccc}
\hline \multicolumn{1}{c}{ Helmintos } & Casos & \multicolumn{5}{c}{ Municípios } & \multicolumn{2}{c}{ Total/Aves } \\
& & Itaboraí & Cambuci & Petrópolis & Correias & S.F.Itabapuana & \\
\hline Ascaridia numidae & No. & $4(66,7)$ & $6(75,0)$ & $2(100)$ & $0(-)$ & $3(75)$ & 15 \\
Paratanaisia bragai & No. & $2(33,3)$ & $2(25)$ & $0(-)$ & $0(-)$ & $0(-)$ & 4 \\
$\begin{array}{l}\text { Raillietina } \\
\text { magninumidae }\end{array}$ & No. & $0(-)$ & $0(-)$ & $0(-)$ & $0(-)$ & $4(100)$ & 4 \\
\hline
\end{tabular}

Tabela 2 - Estado de carne de galinhas-d'angola parasitadas, oriundas de alguns municípios do estado do Rio de Janeiro - 2003

\begin{tabular}{lccccc}
\hline \multicolumn{1}{c}{ Helmintos } & Casos & Bom & $\begin{array}{c}\text { Estado da carne } \\
\text { Regular }\end{array}$ & Rui & Total/Aves \\
\hline Ascaridia numidae & No. & $10(45,4)$ & $0(-)$ & $5(22,7)$ & 15 \\
Tanaisia bragai & No. & $2(9,0)$ & $0(-)$ & $2(9,0)$ & 4 \\
Raillietina & No. & $3(13,6)$ & $1(4,5)$ & $0(-$ & 4 \\
\hline
\end{tabular}

Na necrópsia, foi observada palidez da musculatura peitoral. No trato entérico, o duodeno apresentou mucosas congestas com conteúdo mucoso e exemplares de gênero Ascaridia numidae foram observados no lúmen dos segmentos do jejuno-íleo. Em algumas aves chegavam a obstruir parcial ou totalmente tais estruturas. Nas alterações microscópicas, observou-se uma enterite crônica em 86,7\% com espessamento da mucosa, focos hemorrágicos e um discreto infiltrado inflamatório. As formas larvares foram encontradas inseridas e entre as vilosidades intestinais.

Cestóides da espécie Raillietina magninumida se encontravam fixados à mucosa entérica, evidenciando macroscopicamente uma extensa área congesta com petéquias e conteúdo mucoso. Observaram-se, ainda, nódulos linfáticos aumentados e congestos. As lesões microscópicas foram caracterizadas por uma enterite mucóide com destruição da mucosa, infiltrado inflamatório heterofílico e linfocítico, caracterizando como enterite em $100 \%$ das aves parasitadas por este e por vezes alguns exemplares fixados à mucosa.

Quanto ao gênero Tanaisia bragai, não se observaram alterações macroscópicas. Microscopicamente observou-se nefrite intersticial crônica predominantemente heterofílica acompanhada de fibrose. Os parasitas foram observados em grande quantidade na luz dos ductos coletores que se encontravam bastante dilatados, porém foi encontrada nefrite discreta em $14 \%$ das aves que estavam infectadas por este parasita.

As alterações macroscópicas e microscópicas de cada gênero estudados estão relacionadas na Tabela 3.

Tabela 3 - Alterações macroscópicas e microscópicas observadas por alguns helmintos encontrados em galinhas-d’angola (Numida meleagris L.) procedentes do Estado do Rio de Janeiro - 2003

\begin{tabular}{cccc}
\hline Helmintos & Órgão & \multicolumn{2}{c}{ Alterações } \\
& parasitado & macroscópicas & microscópicas \\
\hline
\end{tabular}

$\begin{array}{cl}\text { Ascaridianumidae } \quad \begin{array}{l}\text { Intestino } \\ \text { delgado }\end{array} & \begin{array}{l}\text { Oclusões parciais ou totais do } \\ \text { íleo e porção final do jejuno, } \\ \text { espessamento, congestão e } \\ \text { focos de hemorragia na } \\ \text { mucosa, exsudato mucoso no } \\ \text { lúmen. }\end{array}\end{array}$

Tanaisiabragai $\quad$ Rim
Raillietinamagninumida Intestino Congestão, presença de delgado petéquias e exemplares fixados.

Discreta reação inflamatória. Presença de larvas inseridas na mucosa e entre as vilosidades intestinais. Presença de heterófilos.

Presença de exemplares no interior dos ductos coletores associados a uma discreta reação inflamatória peritubular.Discreta fibrose. Pronunciada distensão dos ductos.

Enterite crônica. Áreas de destruição da mucosa. Presença de parasitas fixados. 


\section{Discussão e conclusão}

No presente trabalho, foi verificada uma frequência de $77,27 \%$ das aves infectadas, taxa menor em relação ao encontrado por Menezes,1999, em municípios do Estado do Rio de Janeiro, e por Fabiyi, 1972; Hodasi, 1976; Vercruysse et al., 1985, na África, os quais que necropsiaram respectivamente 126 , 45 e 103 galinhas d'angola oriundas de criações extensivas onde encontraram 100\% das aves parasitadas.

Khan e Haziev, 1991, em Bashkir, na antiga União Soviética, constataram que 451 galinhas-d'angola $(82,4 \%)$, de um total de 547 animais, possuíam pelo menos uma espécie de helminto. Por outro lado, Boado et al., 1991, em Cuba, em estudo clínico e anátomo-patológico em 345 galinhasd'angola, chegaram à conclusão de que a enfermidade mais comum nestas aves foram as helmintoses, com prevalência de $69,27 \%$.

Comparando dados desta pesquisa com o de outros autores citados, observa-se que as helmintoses são freqüentes nas criações extensivas de galinha-d'angola.

Tal fato pode ser explicado, não só pela grande quantidade de hospedeiros intermediários de helmintos presentes no meio ambiente das aves, pelo constante contato com o solo contaminado, mas também por serem as galinhas-d'angola mais susceptíveis a estas infecções quando criadas em bandos, mesmo sendo eles caracterizados como criação extensiva.

A maioria das galinhas-d'angola necropsiadas neste estudo abrigavam um grande número de helmintos da espécie $A$. numidae no lúmen do intestino delgado. Em apenas uma das aves observaram-se exemplares no ceco, fato este também registrado por Ruff ( 1991 ); esta ave tinha um grande número de parasitas em todo o segmento intestinal.

As alterações encontradas nas carcaças das galinhasd'angola parasitadas por helmintos e os sinais clínicos observados foram semelhantes aos observados por Orlandi (1969); Macchioni et al. (1978); Boado et al. (1991); Vassilev e Jooste, (1991) e Boado et al. (1991). Macchioni et al. (1978) e Souza et al. (1997) que registraram um quadro de anemia, anorexia, perda de peso, prostração, asas caídas, distrofia óssea, atrofia muscular, palidez das carcaças e diarréia em galinha-d'angola com carga parasitária elevada do helminto A. numidae. Já Boado et al. (1991) relataram sinais clínicos associados a várias espécies de helmintos, caracterizada por palidez, plumagem eriçada, enfraquecimento e retardo no desenvolvimento.

No presente trabalho, não foi possível fazer uma associação entre o quadro clínico apresentado pelas aves parasitadas

\section{Referências}

AMATO, J. F. R.; BOEGER,W. A.; AMATO, S .B. Protocolos para laboratório, coleta e processamento de parasitos de pescado. Itaguaí: U.F.R.R.J., Imprensa Universitária, 1990. 81p.

ARNIZAUT, A. B.; HAYES, L., OLSEN, G. H.; TORRES, J. S.; RUIZ,C. An epizootic of Tanaisia bragai in a captive population of Puerto Rican plain pigeon (Columba inornata wetmorei). Annals of the New York Academy of Sciences, n. 653, p. 202-205, 1992. com uma determinada espécie de helminto presente, uma vez que quase a totalidade das infecções encontradas foram mistas. Avaliação também feita por Menezes, 1999; mas, sem dúvida, a associação destes contribui para o agravamento das lesões causadas.

À necrópsia das aves com elevada intensidade de infecção por $A$. numidae, observou-se um aumento de volume do intestino delgado, espessamento, congestão e focos de hemorragia na mucosa, além de grande quantidade de muco de coloração branco-amarelada na porção dos segmentos do jejuno-íleo. Em apenas uma ave observou-se distrofia óssea do esterno. Estes resultados foram semelhantes aos de Souza et al. (1997). A localização das formas larvares e adultas no presente trabalho estavam de acordo com os achados de Macchioni (1968); Mabon e Reid (1973); Macchioni et al. (1978); Souza et al. (1997); Menezes (1999), como a presença de larvas inseridas na mucosa intestinal, conforme achados de Mabon e Reid (1973), e aos de Menezes (1999), ao estudarem o ciclo dos estágios larvares do $A$. numidae.

Apesar de seu tamanho e sua localização, o trematódeo $P$. bragai, presente na intensidade média de $18,2 \%$, em cada rim das aves parasitadas. Achados semelhantes foram relatados por Santos (1934) em rins de pombos e galinhas domésticas parasitadas por esse helminto, e mais recentemente foi assinalado o primeiro caso deste trematódeo em rolinhacaldo-de-feijão (Columbina talpacoti Temminck, 1811), por Pinto et al. (2003). Assim como no presente estudo, os autores citados encontraram também $P$. bragai na região medular dos rins, mais precisamente no interior de ductos coletores, que se apresentavam dilatados e com paredes espessadas comprimindo os ductos vizinhos em meio a uma reação inflamatória no tecido intersticial e infiltrado inflamatório peritubular predominantemente linfocitário e com alguns heterófilos presentes.

Raillietina magninumida, na intensidade média de infecção $13,6 \%$ encontrada entre as galinhas-d'angola parasitadas na presente pesquisa, demonstrou ser um helminto bastante patogênico, as alterações macroscópicas foram dignas de nota, uma vez que estavam associadas a congestão, petéquias, espessamento, muco e exemplares fixados à mucosa. Em relação à microscopia observou-se destruição da mucosa nos pontos de fixação do parasita.

Apesar da carga parasitária ser baixa, estabelecendo uma comparação entre a ação do parasita e a lesão promovida, convém ressaltar a importância da avaliação descritiva da injúria como um grande fator contribuinte para o agravamento do quadro clínico de cada ave junto às infecções por outros helmintos.

BARGER, E. H.; CARD, L. E.; POMEROY, B. S. Enfermedades y parasitos de las aves. Biblioteca Ténica de Agricultura y Ganadena. México: Union Tipografía Editorial Hispano Americana, 1959. 398 p.

BEHMER, A. O; TOLOSA, E. N. C.; FREITAS, A G. N. Manual Técnico para Histologia_Normal e Patológica. São Paulo: EDART, 1976. 265 p. BOADO, E.;ZALDIVAR, L.; LOPEZ, S.; GONZALES, A.; QUINTERO, D. Diagnostico y_estudio patomorfologico de las enfermidades de la Gallina da Guinea. Revta. Cubana Cienc. Avícola. v. 18, p. 156-161, 1991. 
BORDIN, E.L. ratado de ornitopatologia sistêmica. São Paulo: Nobel, 1981. $210 \mathrm{p}$.

CEDEÑO, H.; MAYAUDON, H. T. Contribuicion al estudio de la fauna parasitaria de las aves de Venezuela (II). Rev. de Med. Vet. Parasitol. Maracay. v. 22, n. 1-8, p. 39-50, 1967-1968.

CHADFIELD, M.; PERMIN, A.; NANSEN P.; BISGAARD, M. Investigation of the parasitc nematode Ascaridia galli (SHRANK, 1788) as a potencial vector for Salmonella enterica dissemination in poultry. Parasitology Resarch. v. 87, n. 4, p. 317-325, 2001.

CHAPMAN, P. A. On a Sex difference in the infection rate of birds with Syngamus trachea. J. Helminth., v. 17, p. 192-194, 1939.

DUARTE, M. J. F. Helmintos parasitos de animais domésticos no Estado do Rio de Janeiro. Arquivos da Escola de Veterinária da UFMG, v. 33, n. 1, p. 67-98, 1981.

EUZÉBY, J. El parasitismo en patología aviar. Zaragoza, España: Editorial Acribia, 1961. $128 \mathrm{p}$.

FABIYI, J.P. Studies on parasites of the grey-breasted helmet guinea fowl (Numida meleagris galeata pallas) of the Vom area of the Benue Plasteau State, Nigeria. I. Helminth parasites. Bulletin of Epizootic Diseases of Africa, v. 20, n. 3, p. 235-238, 1972.

FREITAS, M. G.; COSTA, H. M. A. Raillietina (Paroniella) magninumida Jones, 1930. Arquivos da Escola de Veterinária, Belo Horizonte, v. 14, p. 171-175, 1962.

KHAN, S. A.; HAZIEV, G. Z. Helminths of guinea fowl ( Numida meleagris) in Bashkir ASSR. Veterinary Parasitology. v. 38, p. 349353, 1991.

KING, N. W.; HUNT, R. D.; JONES, T. C. Patologia Veterinária. 6. São Paulo: Manole, 2000.1415 p.

LEVINE, N. D. Nematode parasites of domestic animal and man. Minneapolis: Burguess Publ. Co., 1980. 47 7p.

LÓPEZ, J. T.; AMPARÁN-SALIDO, R. Contribución al conocimiento del parasitismo en_dos especies de aves silvestres de ciudad Universitaria, San Nicolás de los Garza, Nuevo_León, México. Revista Biotam NET, 1999. Acesso em: 8 jan. 2003.

LÚCIO-MARTINÉZ, B. Procedimentos de exame e necrópsia em aves. Departamento de Medicina Veterinária Animal Aviária e Aquática. Faculdade de Medicina Veterinária do Estado de Nova York. Universidade de Cornell, Ithaca, N.Y., 1999. NET. Acesso em 8 jan. 2002.

MABON, J. L.; REID, W. M. Tissue Stages in the Development of Ascaridia numidae from the guinea fowl (Numida meleagris). The journal of Parasitology, v. 59, n. 6, p. 1012-1015, 1973.

MACCHIONI, G. Ascaridia numidae (Leiper, 1908) Travassos, 1913 in guinea fowl (Numida meleagris L.) in Italy. Annali della Facoltá di Medicina Veterinária di Pisa, v. 21, p. 135-143, 1968.

MACCHIONI, G.; MARCONCINI, A.; FUSO, F. Pathogenic effects of $A$ numidae in the guinea fowl. Annali della Facultá di Medicina Veterinária di Pisa, v. 31, p. 115-126, 1978.

MALHOTRA, S. K. Interrelationship of Heterakis pavonis in poultry of na Indian sub-humid region with season, temperature and host Sex. J. Parasit. Appl. Biol., v.1, p. 77-89, 1992.

MATTA, S. C.; AHLUWALIA, S. S. A note on the Pathogenesis of Ascaridia numidae larvae in the gut of guinea fowl. Indian Journal of Animal Sciences, v. 49, n. 1, p. 72-74, 1979.

MENEZES,R. C. Freqüência e patologia das infecções causadas por nematóides e cestóides em galinha-d'angola (Numida meleagris LINNAEUS, 1753) criadas extensivamente no Estado do Rio de Janeiro, Brasil. R. bras. Ci Vet., v. 8, n. 1, p. 35-39, jan./abr. 2001.

MENEZES, R. C.; MATTOS-Jr., D. G.; TORTELLY, R.; MUNIZ-PEREIRA, L. C.; PINTO, R. M.; GOMES, D. C. Trematodes of free range reared guinea fowls (Numida meleagris LINNAEUS, 1758) in the statesof Rio de Janeiro, Brazil: morphology and pathology. Avian Pathology. v. 30, p. 209-214, 2001.
MOND, A.; MALHOTRA, S. K.; CAPOOR, V. N. Maturity status and seasonality of Raillietina (Raillietina) permista (southwell and lake) infesting poultry of an Indian sub-humid region. Bulgarian Academy of Sciences. p. 13 - 20, 2001, NET. Acesso em 15 set. 2003.

NORTON, R. A.; HOPKINS, B. A.; SKEELES, J. K.; BEASLEY, J. N.; KEEGER, J. M. High Mortality of domestic turkeys associated with Ascaridia dissimilis. Avian Diseases, v. 36, p. 469-473, 1992.

NORTON, R. A.; HOERR, I. J.; CLARK, F. D.; RICKE, S. C. Ascarid associated Hepatic foci in Turkeys. Avian Diseases. v. 43, p. 29-38, 1999.

OKAEME, A. N. Disease of young Helmet Guinea fowl (Numida meleagris galeata, PALLAS) under intensive management in Nigéria. Bull. Anim. Heth. Prod. Afr., v. 29, p. 317-319, 1981.

ORLANDI, M. Acuarosis (Dispharagosis) of birds. First report in Italy of Acuarosis (Dispharogosis) by Dispharynx nasuta (Rudolphi, 1819) In the guinea-fowl (Numida meleagris L.). Annali della Facoltá di Medicina Veterinária di Pisa. v. 32, p. 105-110, 1979.

ORTIZ, J. Prevencion y Control de Parasitos Internos en Ponedoras Comerciales. Asociación de Avicultores de Santa Cruz, Santa Cruz de la Sierra, Bolívia. ADA, 2000, NET. Acesso em: 8 jan. 2003.

PINTO,C. Zoo-Parasitos de Interesse Médico Veterinário. 2. ed. Rio de Janeiro: Editora Scientífica, 1945. 461 p.

RANDALL, C. Diseases and disorders of the Domestic Fowl and Turkey. 2nd ed. London, England: Mosby: 1991. 175 p.

REID, W.M. Chicken and Turkey Tapeworms: Handbook to aid in the identification and control of tapeworms found in the United States of America. EUA: University of Georgia Poultry Department, 1962. 71 p.

REID, W.M.; MABON, J. L. Tissue stages in the developtment of Ascaridia numidae from the ginea fowl (Numida meleagris). The journal of Parasitology, v. 59, n. 6, p.1012-1015, 1973.

ROSSKOPF, W; WOERPEL, R. Diseases of Cage and Aviary Birds. $3^{\text {rd }}$ Baltimore, USA: Williams \& Wilkins, 1996, $1088 \mathrm{p}$.

RUFF, M. D. Nematodes and Acanthocephalans. In: CALNEK, B. W.; BARNES, H.; REID, W. M. et al. Diseases of Poultry. 9. ed. EUA: lowa State University Press, 1991.929 p. Cap. 31, p. 731-763.

SANTOS, V. Monostomose renal das aves domésticas. Revista do Departamento Nacional de Produção Animal. v.1, p. 203-211, 1934.

SHEVTSOV, A. A.;ZASKIND, L. N.; PILIPCHUK, R. I.; POZHIVAL, A. I. Some aspects of the epizootiology of Ascaridia infection in Turkeys. In: Prob. Parazit. "Nauk Dumka”, 1975, p. 268-269.

SOUZA, P. C. A.; RODRIGUES, M. L. A.; LOPES, C. W. G. Um surto de Ascaridiose em uma criação intensiva de galinhas-d'angola. Revista Brasileira de Medicina Veterinária, v. 19, n. 5, p. 206-208, 1997.

SPÍNOLA, R. Consumo de galinha d'angola deve ser popularizado. $O$ Estado de S. Paulo. NET ESTADO, Jun.1997. Acesso em: 8 jan. 2003.

TARBES, H. M.; CEDEÑO, H. Contribución al Estudio de la Fauna Parasitaria de las aves de Venezuela (II). Com la descripción de cuatro nuevas especies para Venezuela. Revista de Medicina Veterinaria y Parasitologia Maracay, Venezuela, v. 22, n. 1-8, p. 39-50, 1967-1968. THATCHER, E.V. Trematódeos Neotropicais. Manaus: INPA, 1993, $553 \mathrm{p}$.

TRAVASSOS, L.; FREITAS, J.F.T.; KOHN, A. Trematódeos do Brasil. Memórias do_Instituto Oswaldo Cruz, Rio de Janeiro, v. 67, p. 1-886, 1969.

UENO \& GONÇALVES, P. C. Manual para Diagnóstico das Helmintoses de Ruminantes. JICA, TÓKYO, 165 p. 1994.

UMECHE, A.; ENO, R. O. A. A survey of parasites of chickens from poultry farms in Calabar, Nigéria. Revista Latino- americana Microbiol., v. 29, p. 133-136, 1987.

URQUHART, G. M.;ARMON, J.; DUNCAN, J. L.; DUNN, A. M.; JENNINGS, F. W. Parasitologia Veterinária. Rio de Janeiro: Guanabara Koogan, 1990. 
VASSILEV, G. D.; JOOTSE, R. Pathology and Taxonomy of Synhimantus (Dispharynx) nasuta infesting bantanms and guinea-fowls in Zimbabwe. Bulletin of animal Health and production in Africa, v. 39, n.1, p. 27-30, 1991.

VON BRAND, T. Biochemistry of Parasites. $2^{\text {nd }}$. NY: London: Acad. Pr., 1973.

VERCUYSSE, J. ; HARRIS, E. A; BRAY, R. A. et al. A survey of Gastrintestinal helminthes of the Common Helmet Guinea Fowl (Numida meleagris galeata) in Burkina Faso. Avian Diseases, v. 29, n. 3, p. 742-745, 1985.

VICENTE, J. J.; RODRIGUES, H. O.; GOMES, D. C.; PINTO, R. M. Nematóides do Brasil. Parte IV: Nematóides de aves. Revista Brasileira de Zoologia, v. 12 (supl. 1), p. 1-273, 1995.
WARDLE, R. A.; Mc LOAD, J. A. The Zoology of Tapeworms. Minneapolis: Univ. of Minnesota Press, 1952. 780 p.

WILLOUGHBY, D. H.;BICKFORD, A. A.; CHARLTON, B. R.; COOPER, G. L.; LINARES, J. A. Ascaridia dissimilis larval migration associated with enteritis and low market weights in meat Turkeys. Avian Diseases. v. 39, p. 837-843, 1995.

YAMAGUTI, S. The Nematodes of Vertebrates: Systema Helminthum. New York, Interscience, 3 v., part I e II, 1961. 1261 p.

ZANDER, D.V.; MALLINSON, E. T. Principles of disease Prevention: diagnosis and control. In: Calnek, B. W.; BARNES, H. J.; REID, W. M. et al. Diseases of poultry. 9 ed. EUA: Iwoa State University Press, 1991. p. 3-44. 